\section{IN THE NEWS}

\section{Branding powe}

A preference for the taste of Coke or Pepsi lies as much in the mind as on the tongue, according to a study led by Read Montague at Baylor College of Medicine. “Everybody's heard of Coke and Pepsi. They have messages and, in the case of Coke, those messages have insinuated themselves in our nervous system," he told BBC News Online (13 October).

Everybody knows the power of brand marketing, but no one has previously shown that it has a direct effect on the brain's capacity to make a choice. The researchers decided to use Coke and Pepsi because, although the two drinks are almost identical both chemically and physically, people often strongly favour one over the other.

Volunteers were given a blind taste test, during which their brains were scanned using functional MRI. Both drinks evoked brain responses in the ventromedial prefrontal cortex.

When subjects saw a picture of a can of Coke before their sips, other parts of the brain, including dorsolateral prefrontal cortex and the hippocampus, were also activated. "These regions are thought to be involved in recalling culture influences and modifying behaviours based on emotion and mood." (BBC News Online).

Intriguingly, this did not occur when they saw a Pepsi can. The study "demonstrates in stark neurological terms the way in which brand images and aggressive marketing sway choices made by the brain." (The Times, UK,

14 October).

The findings are not mere academic curiosity. The researchers believe that they are "particularly important given the role that sugared soft drinks have on the epidemic of childhood obesity and type 2 diabetes." (The Independent, UK, 17 October).

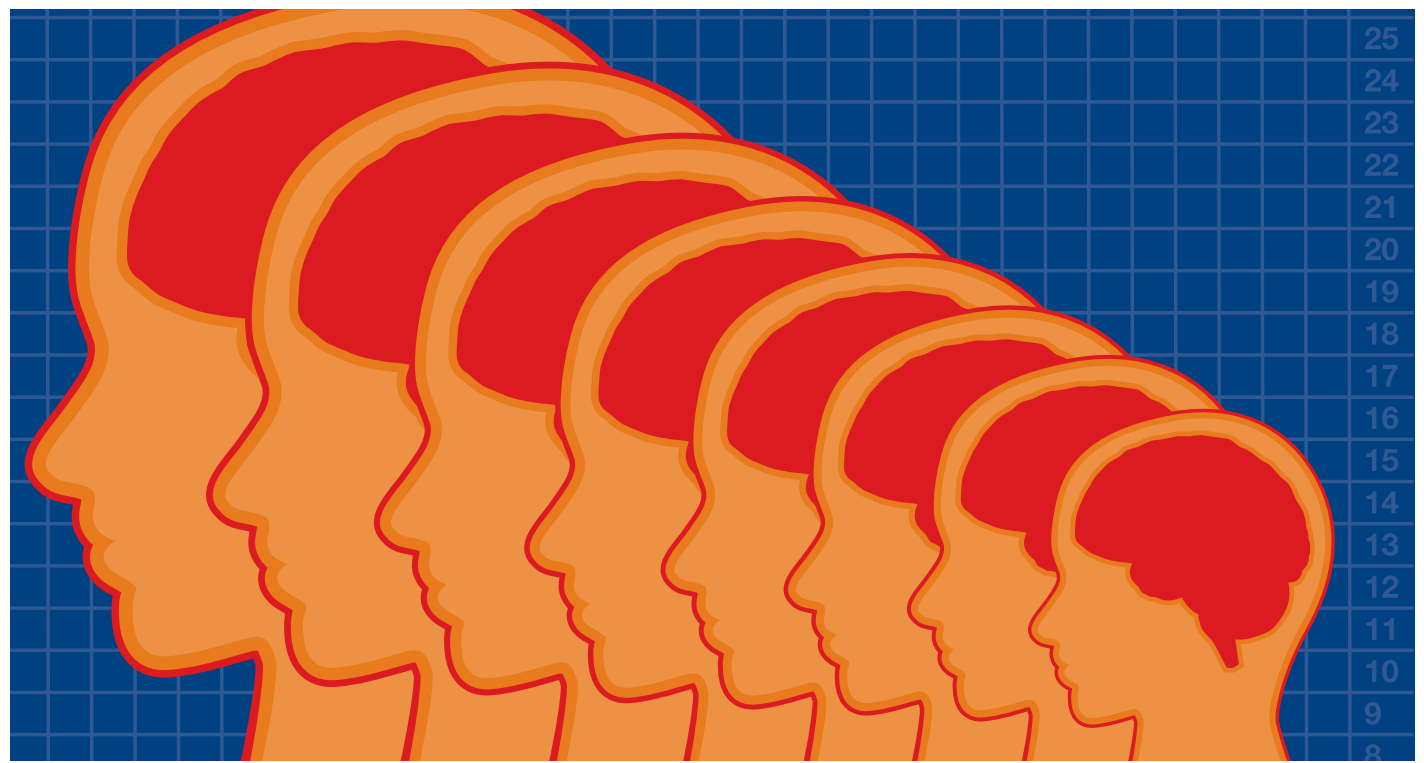

NEUROGENESIS

\title{
Sizing up the CNS
}

A fundamental issue in developmental neuroscience is how the requisite numbers of neurons and glia are generated at the appropriate times, so that the CNS attains its correct size and shape. Reporting in Development, Hatakeyama and colleagues present evidence that the Hes family of basic helix-loop-helix factors has a pivotal role in regulating the size and cellular composition, and even the integrity, of the CNS.

The earliest stem cells in the neural tube are neuroepithelial cells, which proliferate through a series of symmetrical divisions. In mice, at about embryonic day (E) 8.5, these cells begin to take on the characteristics of radial glia. During the ensuing phase of neurogenesis, the radial glial cells divide asymmetrically to generate neurons and more radial glia.

The Hes 1 and Hes5 genes code for effectors of the Notch signalling pathway, and they are expressed extensively in the embryonic CNS. Previous studies have indicated that they are required for the maintenance of neural stem cells, but single knockouts of either gene produce only mild phenotypes, perhaps because they can partially compensate for one another. Consequently, Hatakeyama et al. decided to examine the effects of inactivating both genes.

In Hes 1;Hes 5 double mutants, neuroepithelial cells developed normally at first and began to generate radial glia. However, by E9.5, many of the radial glia in the ventral neural tube had been replaced by spherical cells that expressed neuron-specific markers, and by E10.5 (the time at which the embryos began to die), the dorsal neural tube was also affected. There was no evidence of increased cell death, so the authors concluded that the radial glia had differentiated prematurely into neurons. The hindbrain and spinal cord were most severely affected, but defects were also observed in more anterior regions; for example, the optic vesicles failed to develop in the forebrain.

The apical junctions and basal lamina of the neural tube were also disrupted in the Hes1;Hes5 mutants, allowing cells to escape into surrounding tissue or the neural tube lumen. Ablation of radial glia in wild-type embryos had a similar effect, indicating that the radial glia are required to maintain the integrity of the inner and outer walls of the CNS.

As neuroepithelial cells initially develop normally in the Hes1;Hes5 double mutants, Hatakeyama et al. wondered whether another Hes gene might compensate for loss of function of these genes at early stages. A strong candidate was the Hes3 gene, which is expressed in the embryonic CNS as early as E8.0. In Hes1;Hes3;Hes5 triple mutant embryos, neuroepithelial cells were generated, but their stem-cell properties were not maintained, and many had differentiated into neurons by E8.5. At E9.5, most of the cells in the ventral spinal cord had differentiated into neurons, and virtually all of the radial glia had become neurons by E10.0.

Hatakeyama et al. propose that Hes1 and Hes3 are required to maintain the neuroepithelial cell population, and that Hes 1 and Hes 5 (and perhaps Hes 3 to some extent) are responsible for maintaining radial glia. Radial glia have to remain in a neurogenic state for a defined period to ensure that appropriate numbers of neurons are produced, after which they usually switch to a gliogenic state. In the absence of Hes gene function, the supply of stem cells is exhausted long before this switch can be made.

Heather Wood

(2) References and links

ORIGINAL RESEARCH PAPER Hatakeyama, J. et al. Hes genes regulate size, shape and histogenesis of the nervous system by control of the timing of neural stem cell differentiation. Development 131, 5539-5550 (2004) 